\title{
CODIFIER LE DIVORCE INTERNATIONAL QUELQUES REMARQUES SUR LE PROJET GEDIP
}

\author{
ETIENNE PATAUT*
}

\begin{abstract}
Codifying international divorce. Some remarks about the GEDIP project
The EU legislator has modified once again the so-called Brussels 2 bis Regulation. The new regulation 2019/1111 adds new provisions on parental responsibility and child abduction, but leaves those on divorce largely untouched. To the contrary, the GEDIP, an expert group of academics in private international law from across Europe, has established an indepth proposal for a new regulation on divorce. This paper analyzes the proposal which seeks to modernize and improve the current situation that has been widely criticized. The proposed regulation covers choice of law, jurisdiction and recognition. It allows limited party autonomy, both in jurisdiction and choice of law, and suggests significant improvements of the provisions on objective jurisdiction. It also recommends a recognition mechanism that would apply to judgements from third States and provides for a comprehensive set of rules concerning private divorces.
\end{abstract}

Keywords: divorce; choice of laws; jurisdiction; recognition; GEDIP

Mots clés : divorce ; conflits de lois ; compétence ; reconnaissance ; GEDIP

DOI: $10.14712 / 23366478.2020 .34$

Le droit international privé de la famille est en complet bouleversement en Europe. Deux facteurs, différents et indépendants, y contribuent.

Le premier est la jurisprudence de la Cour de justice en matière de liberté de circulation et de citoyenneté européenne. A partir de son interprétation extensive de la notion de citoyenneté et au fil d'une jurisprudence audacieuse, la Cour a progressivement posé des règles importantes et libérales, en matière, par exemple, de nom de famille, ${ }^{1} \mathrm{de}$ mariage $^{2}$ ou de kafala. ${ }^{3}$ Toutes ces règles dévoilent peu à peu un schéma d'ensemble,

* Ce texte a bénéficié de la lecture critique et amicale de M. Fallon et C. Kohler, qui l'ont considérablement amélioré ; qu'ils en soient chaleureusement remerciés. Toutes les erreurs éventuelles, bien entendu, sont de mon seul fait.

Etienne Pataut - Professeur à l'École de droit de la Sorbonne, membre du Groupe européen de droit international privé.

1 En dernier lieu : CJUE, 8 juin 2017, aff. C-541/15, Freitag.

2 CJUE, Gde Chambre, 5 juin 2018, aff. C-673/16, Coman.

3 CJUE, Gde Chambre, 26 mars 2019, aff. C-129/18, SM. 
méthodologiquement orienté autour de la théorie de la reconnaissance 4 et qui s'insère progressivement dans le droit international privé des États membres.

Le second facteur, beaucoup plus évident à identifier, est l'importance de l'œuvre législative européenne en matière de droit de la famille. A partir de la Convention dite de Bruxelles 2,5 inspirée par un projet du Groupe européen de droit international privé ${ }^{6}$ et jamais entrée en vigueur, ont été adoptés plusieurs règlements importants en matière de droit de la famille. La Convention initiale a ainsi été transformée en règlement européen, ${ }^{7}$ lui-même modifié ${ }^{8}$ puis encore modifié. ${ }^{9}$ Ensuite, à ce texte relatif au divorce, à la responsabilité parentale et à l'enlèvement d'enfants ont été ajoutés d'autres règlements, sur la loi applicable au divorce ${ }^{10}$ et sur les obligations alimentaires, les successions et les régimes matrimoniaux. ${ }^{11}$

Pour l'essentiel, ces règlements restent de facture relativement classique, en ce sens qu'ils posent des règles de conflit, de compétence internationale et de reconnaissance des décisions qui, pour être modernisées et nouvelles, n'en appartiennent pas moins à la tradition du droit international privé traditionnel.

Deux lignes d'évolution, donc, et, à cet égard, l'un des défis majeurs de la discipline sera de réunir ces deux fils pour permettre la naissance d'un véritable droit international privé européen de la famille, fondé sur des objectifs politiques et des bases juridiques propres à l'Union. ${ }^{12}$

${ }^{4}$ LAGARDE, P. La reconnaissance est-elle l'avenir du droit international privé? Rec. Cours, 2015, vol. 371, p. 9.

5 Convention concernant la compétence, la reconnaissance et l'exécution des décisions en matière matrimoniale du 28 mai 1998, JOCE, no C 221 du 16 juillet 1998.

6 Groupe européen de droit international privé, « Proposition pour une convention concernant la compétence judiciaire et l'exécution des décisions en matière familiale et successorale », Réunion de Heidelberg de 1993, texte à la Rev. Crit. DIP, 1993, 841 ; sur de texte, v. GAUDEMET-TALLON, H. « La Convention dite "Bruxelles II" : convention concernant la compétence, la reconnaissance et l'exécution des décisions en matière matrimoniale ». Trav. Com. fr. DIP, 1998-2000, p. 83.

7 Règlement $\mathrm{n}^{\circ}$ 1347/2000, relatif à la compétence, la reconnaissance et l'exécution des décisions en matière matrimoniale et de responsabilité parentale des enfants communs, JOCE $\mathrm{n}^{\circ} \mathrm{L} 160$ du 30 juin 2000, dit Règlement Bruxelles 2.

8 Règlement no 2201/2003, relatif à la compétence, la reconnaissance et l'exécution des décisions en matière matrimoniale et en matière de responsabilité parentale, JOCE $\mathrm{n}^{\circ} \mathrm{L} 338 \mathrm{du} 23$ décembre 2003, dit règlement Bruxelles 2 bis.

9 Règlement 2019/1111, relatif à la compétence, la reconnaissance et l'exécution des décisions en matière matrimoniale et en matière de responsabilité parentale, ainsi qu'à l'enlèvement international d'enfants, JOUE $\mathrm{n}^{\circ} \mathrm{L} 178$ du 2 juillet 2019, dit règlement Bruxelles 2 ter.

10 Règlement $\mathrm{n}^{\circ}$ 1259/2010 du 20 décembre 2010 mettant en cuvre une coopération renforcée dans le domaine de la loi applicable au divorce et à la séparation de corps, JOUE $\mathrm{n}^{\circ} \mathrm{L} 343$ du 29 décembre 2010, p. 10 (dit Règlement Rome 3).

11 Respectivement : Règlement 4/2009 du 18 décembre 2008 relatif à la compétence, la loi applicable, la reconnaissance et l'exécution des décisions et la coopération en matière d'obligations alimentaires, JOUE, $\mathrm{n}^{\circ}$ L. 7 du 10 janvier 2009, p. 1 ; Règlement no 650/2012 du 4 juillet 2012 relatif à la compétence, la loi applicable, la reconnaissance et l'exécution des décisions, et l'acceptation et l'exécution des actes authentiques en matière de successions et à la création d'un certificat successoral européen, JOUE $\mathrm{n}^{\circ} \mathrm{L}$ 201 du 27 juillet 2012, p. 107 ; Règlements 2016/1103 et 2016/1104 du 24 juin 2016 mettant en cuvre une coopération renforcée dans le domaine de la compétence, de la loi applicable, de la reconnaissance et de l'exécution des décisions en matière de régimes matrimoniaux (ou, pour le second, d'effets patrimoniaux des partenariats enregistrés), JOUE $\mathrm{n}^{\circ}$ L 183 du 8 juillet 2016, p. 1.

12 RASS-MASSON, L. The Foundations of European Private International Family Law. Yearbook of Private International Law, 2018/2019, Vol. 20, pp. 217-231. 
En attendant, chacun des grands domaines du droit de la famille évolue peu à peu, au fur et à mesure des textes et des arrêts.

A cet égard, le droit du divorce international est le grand oublié de la réforme du règlement Bruxelles 2 bis. ${ }^{13}$ Le nouveau texte ne prévoit en effet guère de modification fondamentale, à l'exception d'une importante et discutable règle nouvelle sur les divorces sans juge, on y reviendra. Les critiques, pourtant, ne manquent pas. Les règles de compétence, surtout, sont vigoureusement discutées en raison de leur complexité et de leur nombre, véritable appel au forum shopping. ${ }^{14}$ Mais tout autant, les critiques n'épargnent parfois pas non plus les règles de conflit de lois et la place qu'elles laissent à l'autonomie de la volonté, ou même, les règles de reconnaissance et d'exécution, notamment en raison de la politique substantielle qu'elles véhiculent. ${ }^{15}$ Toutes ces règles, donc, vont rester inchangées jusqu'à un incertain règlement Bruxelles 2 quater ou Rome 3 bis, bien improbables à court terme.

Le choix est certes regrettable. Il ne doit pas pour autant empêcher la réflexion d'avancer en la matière. Si le droit du divorce paraît être parvenu à un certain équilibre, en effet, cet équilibre reste instable et critiqué et pourrait être amélioré. A cet égard, la proposition faite par le Groupe européen de droit international privé d'un nouveau règlement en matière de divorce est un jalon suffisamment important pour mériter une analyse plus approfondie. ${ }^{16}$

La proposition de texte offre en effet à la réflexion quelques règles plus ou moins nouvelles selon les cas, en matière de compétence (2), de loi applicable (3) et de reconnaissance (4). Il faudra toutefois dire un mot du champ d'application (1) et isoler la question, difficile entre toutes, du divorce sans juge (5).

\section{CHAMP D’APPLICATION}

\section{A) QUESTIONS DE MÉTHODE}

La première remarque à faire est méthodologique. Comme on le sait, deux modèles, en matière de droit international privé de l'Union, s'opposent. Le premier, celui des règlements dit « Rome » $(1,2$ et 3$)$ ou « Bruxelles » (1 et 2, dans leurs différentes mues) consiste à unifier les règles de conflit de lois, dans le premier cas, de droit

13 FRANCQ, S. Réforme avortée et réforme surprise : compétence et reconnaissance en matière de dissolution du mariage après la refonte du règlement Bruxelles Iibis. Dans : FRANCQ, S. - SAROLEA, S. (dir.). Actualités européennes en droit familial international. Anthemis, 2019, p. 53.

14 Pour un panorama complet v. part. BONOMI, A. La compétence internationale en matière de divorce. Quelques suggestions pour une (improbable) révision du règlement Bruxelles 2 bis. Revue Critique de Droit International Privé, 2017, pp. 511-534, et KRUGER, T. - SAMYN, L. Brussel 2 bis : successes and suggested improvements. Journal of Private International law, 2016, 1.

15 McELEAVY, P. Integrating the Brussels II bis Regulation in the United Kingdom. Dans : BOELEWOELKI, K. - BEILFUSS, C. G. (eds.). Brussels II bis : its impact and application in the member states. Intersentia, 2007, p. 309 ; ANCEL, B. - MUIR WATT, H. La désunion européenne : le règlement dit Bruxelles 2. Revue Critique de Droit International Privé, 2001, pp. 403-457.

16 GEDIP, proposition de règlement relatif à la compétence, la loi applicable et la reconnaissance en matière de divorce, adopté à la session de Katowice en 2019, disponible sur le site internet : https://www .gedip-egpil.eu/reunionstravail/2019_Katowice/divorce/Divorce-Txt-Final-FR.pdf. 
judiciaire international, dans le second. La seconde méthode est méthodologiquement plus globale mais substantiellement plus étroite. Elle consiste à isoler une question particulière et à en traiter l'ensemble des conséquences internationales. C'est la voie suivie par les grands règlements récents de droit de la famille : successions, régimes matrimoniaux et obligations alimentaires.

C'est aussi la méthode suivie par le GEDIP, qui propose donc d'abandonner, en matière de droit de la famille, la séparation entre conflit de lois et droit judiciaire, pour l'aborder dans un seul texte. L'avantage visé est celui de la cohérence d'ensemble en matière de divorce. L'inconvénient corrélatif est de perdre les liaisons qui peuvent exister avec d'autres questions proches mais tout aussi essentielles.

A cet égard, il ne faut pas se dissimuler une faiblesse majeure du projet GEDIP : celle de ne traiter que du principe du divorce.

Le divorce est aujourd'hui admis, avec plus ou moins de restriction, dans tous les pays de l'Union européenne. Dès lors, en permettre le prononcé et en assurer la circulation est bien évidemment toujours utile, notamment si l'on souligne que le texte concernera aussi les États tiers, mais ce texte n'en reste pas moins peut-être d'une urgence moindre qu'à une époque où les extrêmes différences entre États, y compris européens, en la matière, rendaient d'une particulière importance l'adoption de règles claires et prévisibles.

Aujourd'hui, le contentieux du divorce et post-divorce est très largement un contentieux patrimonial ou portant sur la responsabilité parentale, soit sur des questions qui relèvent d'autres règlements de l'Union. Dès lors, prévoir des règles précises mais portant uniquement sur le principe du divorce s'expose à deux objections. D'une part, le caractère très étroit de son champ d'application en diminue l'intérêt; d'autre part, et surtout, l'adoption de règles particulières à la question du principe du divorce va nécessairement poser une difficulté importante de cohérence d'ensemble. Des époux qui divorcent veulent certes rompre leur lien matrimonial, mais ils veulent aussi régler leurs intérêts pécuniaires et la responsabilité parentale. Éclater les règles en la matière dans différents règlements, c'est risquer l'incohérence, les difficultés d'articulation et les situations boiteuses, source d'importantes difficultés pratiques pour les premiers concernés : les époux et les enfants. ${ }^{17}$ L'empilement de textes pose d'importantes difficultés d'articulation, qui ne seront pas résolues par l'adoption d'un règlement particulier sur le principe du divorce.

L'objection n'est certes pas nouvelle; mais elle n'est non plus pas aggravée par le projet GEDIP, puisque les textes actuels sont déjà limités au principe du divorce. Une éventuelle solution, toutefois, dépasserait le cadre limité de cette contribution. Elle ne pourrait en effet passer que par une véritable codification d'ensemble du droit international privé européen (ou, au moins, du droit international privé européen de la famille) qui tiendrait compte, bien entendu, de l'acquis législatif, mais qui permettrait aussi d'intégrer les solutions désormais imposées par la Cour de justice. On en est loin.

17 Sur ce point, v. particulièrement BOICHE, A. Pratique judiciaire des règlements européens en droit de la famille. Travaux du Comité français de droit international privé 2014-2016. Pedone, 2016, p. 17. 
Autre difficulté : celle du champ d'application matériel du texte. La difficulté n'est pas tellement celle de l'inclusion du divorce entre personnes de même sexe, qui semble inévitable en raison de l'expansion de cette forme de mariage. Bien entendu, nul n'ignore les difficultés politiques qu'engendre une telle inclusion. Il reste que ces difficultés relèvent de la sphère de la décision politique et non de celle du droit, le mariage homosexuel n'étant en rien différent d'un mariage hétérosexuel. Dès lors, la décision de l'inclure s'imposait d'elle-même.

Plus délicate, en revanche, était l'inclusion ou pas de l'annulation du mariage. A l'instar de la séparation de corps, qui entre progressivement dans l'ombre, le contentieux de la nullité du mariage semblait voué à une relative marginalisation en raison de la libéralisation du divorce en Europe. Quel intérêt, en effet, que l'annulation du mariage dans un monde où le divorce est si aisément accessible ?

Deux faits, pourtant, ont démenti ce pronostic hâtif. La lutte contre l'immigration, tout d'abord, a conduit à un renforcement de l'arsenal à la disposition des autorités publiques visant à remettre en cause les mariages de complaisance. ${ }^{18}$ Les exemples en matière de nullité du mariage ont désormais quitté les rives rassurantes et anecdotiques des mariages morganatiques et des virginités douteuses pour aborder ceux, beaucoup plus sinistres, de l'intrusion de la puissance publique au sein même de la liberté du mariage. ${ }^{19}$

D'autre part, la crise causée par les guerres au Moyen Orient et, en particulier, en Syrie, a conduit à un certain renouveau de la question de la nullité des mariages, notamment dans le but de lutter contre les mariages contractés entre ou avec des enfants. L'Allemagne et la Suède, tout particulièrement, ont adopté des règles particulières en la matière, d'ailleurs pas exemptes de toute critique. ${ }^{20}$ Les questions de droit international privé liées à la nullité du mariage ont donc évolué, mais nullement disparu.

Les règlements européens actuels ont sur ce point des positions différentes. Le droit judiciaire inclut le contentieux de la nullité du mariage ${ }^{21}$; le conflit de lois l'exclut. C'est cette seconde solution qui a été préférée dans le projet GEDIP, sous réserve d'une éventuelle question préalable. ${ }^{22}$

18 En France, v. CORNELOUP, S. « Maîtrise de l'immigration et célébration des mariages ». Mélanges P. Lagarde. Dalloz, 2005, p. 207.

19 En matière de nullité du mariage, « le principal demandeur est le procureur de la République qui assigne le couple dans près de six affaires sur dix $(57 \%)$. Le plus souvent, le mariage scelle l'union de conjoints de nationalités différentes $(86,8 \%)$, les couples d'étrangers de même nationalité et de Français étant peu nombreux (respectivement $8,0 \%$ et 5,2 \%) » : LAMARCHE, M - LEMOULAND, J.-J. Mariage : sanctions de l'inobservation des conditions de formation. Répertoire Dalloz de droit civil, 2014 et 2019, no 7.

20 Pour l'Allemagne, v. KOHLER, C. La nouvelle législation allemande sur le mariage et le droit international privé. Revue Critique de Droit International Privé, 2018, pp. 51-58 ; Pour la Suède, v. part. BOGDAN, M. Some critical comments on the new Swedish rules on non-recognition of foreign child marriages. Journal of Private Internatonal Law, 2019, pp. 247-256.

21 CJUE, 13 octobre 2016, aff. C-294/15, Edyta Mikołajczyk c. Marie Louise Czarnecka.

22 L'article $1 \S 3$ du projet précise en effet qu' « un litige n'est pas exclu du champ d'application du règlement lorsqu'une matière exclue en vertu de ce paragraphe est soulevée seulement à titre préalable, notamment à titre de défense ou de demande reconventionnelle». 
$\mathrm{Si}$, en effet, il n'est pas inconcevable de traiter une action ou une décision en matière de nullité du mariage comme une demande ou une décision de divorce au stade de la compétence juridictionnelle ou de la circulation des décisions, l'assimilation est beaucoup plus difficile au stade de la détermination de la loi applicable. Divorce et nullité du mariage sont conceptuellement deux choses bien différentes sous l'angle du droit interne et, partant, sous l'angle des qualifications traditionnelles du droit internationale privé. Le premier s'est progressivement entièrement émancipé de la catégorie « mariage », la seconde, par nature, y appartient pleinement. La loi applicable est donc nécessairement différente. Un règlement unique, sur le modèle des successions ou des régimes matrimoniaux, devait donc ou bien prévoir un double régime de conflit de lois, ajoutant de la complexité à l'ensemble, ou bien exclure la nullité du mariage du champ d'application. C'est, pour d'évidentes raisons de simplicité, ce second choix qui a été fait.

L'exclusion serait évidemment regrettable en ce qu'elle pourrait constituer une régression par rapport à l'état du droit positif qui a permis l'adoption de règles communes en matière de nullité du mariage. L'objection, toutefois, ne fait que renforcer la nécessité d'une refonte plus globale des règles de droit international privé européen de la famille qui prévoirait aussi des règles propres au mariage.

\section{LES RÈGLES DE COMPÉTENCE}

Incontestablement, les règles de compétence sont celles qui ont fait l'objet de l'attention la plus grande, tant les critiques semblent unanimes à l'encontre des solutions du droit positif européen et les propositions de réformes ne manquent pas. ${ }^{23}$

Le projet GEDIP propose de réformer en profondeur la compétence internationale en matière de divorce pour répondre à ces critiques. Pour ce faire, deux modifications fondamentales sont soumises à la discussion. La première, sans doute la plus évidente, concerne les critères de compétence et leur hiérarchisation ; la seconde, les fors exorbitants et les liens avec les pays tiers.

\section{A) CRITÈRES DE RATTACHEMENT ET HIÉRARCHISATION DES RÈGLES DE COMPÉTENCE}

L'article 3 du règlement Bruxelles 2 bis fait sans doute partie des règles les plus unanimement critiquées du règlement. ${ }^{24} \mathrm{Il}$ prévoit, rappelons-le brièvement, un ensemble de compétences en matière de divorce, fondées sur une longue liste de critères qui, tous, tournent autour de la résidence habituelle des époux ou de leur nationalité. Sont ainsi compétentes les juridictions de : la résidence des époux ; la dernière résidence

23 V. not. BONOMI, op. cit., p. 511.

24 GONZALEZ BEILFUSS, C. Jurisdiction Rules in matrimonial Matters under Regulation Brussels 2 bis. Dans : FULCHIRON, H. - NOURISSAT, C. (dir.). Le nouveau droit communautaire du divorce et de la responsabilité parentale. Dalloz, 2005, p. 55 ; dans le même sens, BONOMI, op. cit. ; KRUGER SAMYN, op. cit., pp. 10 et seq. 
habituelle des époux dans la mesure où l'un d'eux y réside encore ; la résidence habituelle du défendeur ; en cas de demande conjointe, la résidence habituelle de l'un ou l'autre époux ; la résidence habituelle du demandeur s'il y a résidé depuis au moins une année immédiatement avant l'introduction de la demande ; la résidence habituelle du demandeur s'il y a résidé depuis au moins six mois immédiatement avant l'introduction de la demande et s'il est ressortissant de l'État membre en question; la nationalité des deux époux (à la nationalité pouvant être substituée le domicile dans le cas du Royaume-Uni et de l'Irlande).

Chacun de ces critères peut être discuté : si certains semblent incontestables (la résidence commune ou la nationalité commune, par exemple), d'autres peuvent être plus critiquables (la résidence de l'époux demandeur, notamment). Mais, surtout, ce qui semble le plus regrettable est l'absence de hiérarchie entre tous ces critères. Tous ces tribunaux sont en effet simultanément disponibles, multipliant, ce faisant, les risques de forum shopping ${ }^{25}$ et, combinés avec la règle rigide de litispendance, de course au for. ${ }^{26}$ La multiplication des règles de compétence accroît, qui plus est, le risque de dissociation entre le for compétent pour le divorce et celui qui aura à connaître des effets du divorce.

Toutes ces critiques, ici fort sommairement résumées, sont bien connues. La décision n'en a pas moins été prises de ne pas y répondre lors de l'adoption du règlement Bruxelles 2 ter en juillet 2019, puisque le nouveau texte reprend, presque à l'identique, l'ancienne disposition de l'article 3. C'est sans doute l'un des aspects les plus regrettables du nouveau règlement.

L'un des objectifs du projet GEDIP, en revanche, est précisément de répondre à cette difficulté, c'est l'objet, plus précisément, des articles 5 et 6 .

Tout d'abord, en effet, les règles de compétence ordinaires prévoient désormais une échelle de chefs de compétence en cascade, dont l'objectif est de lutter contre l'éclatement du contentieux. Le premier choix a été de favoriser le rattachement à la résidence habituelle des époux par rapport à la nationalité de ceux-ci. En matière de statut personnel, le rattachement à la résidence habituelle (notamment sous l'influence des conventions de La Haye) devient progressivement le rattachement de principe, le projet GEDIP s'inscrit nettement dans cette tendance. Surtout, les rattachements sont hiérarchisés. La technique est ici celle des rattachements en cascade : les critères de compétence sont strictement conditionnés et ce n'est que si le premier n'est pas disponible que le deuxième est ouvert, et ainsi de suite. Dans ce cadre, les hypothèses de conflits de compétence sont éliminées, sauf éventuelle discussion sur la localisation de la résidence habituelle, bien sûr.

Ensuite, deuxième innovation, est insérée à l'article 5 la possibilité de conclure une clause attributive de juridiction. L'expansion progressive de l'autonomie de la volonté en matière de droit international privé de la famille n'est plus à démontrer et elle avait d'ailleurs déjà trouvé sa place dans le règlement Rome 3 en matière de loi applicable, on le verra. La possibilité d'une clause attributive de juridiction en la matière permet de fixer par avance le tribunal compétent, apportant peut-être un élément de certitude qui

25 V. p. ex. CJUE, 16 juillet 2009, aff. C-168/08, Haddadi.

26 V. p. ex. CJUE, 16 juillet 2015 , aff. C-184/14, $A$. 
fait gravement défaut dans le texte actuel. L'équilibre choisi, à nouveau relativement classique en droit international privé de la famille, ${ }^{27}$ utilise une technique d'encadrement de l'autonomie de la volonté, puisque le choix ne peut porter que sur les tribunaux du domicile ou de la nationalité. Il reste que les considérations de déséquilibre et de protection ne sont pas entièrement absentes du contentieux du divorce, aussi le troisième paragraphe de l'article 5 permet-il au juge d'écarter la clause si celle-ci s'avère manifestement déraisonnable ou inéquitable à l'égard d'une partie.

Il faut enfin mentionner l'existence à l'article 10 d'une règle de transfert de compétence inspirée par l'article 15 du règlement 2201/2003, devenu 12 du règlement 2019/1111.

L'équilibre proposé est donc bien fondamentalement différent de celui qui existe actuellement dans le règlement Bruxelles 2 bis. Il y a là, sans doute, l'une des suggestions les plus importantes du projet, tant l'état du droit positif est peu convaincant. Mais la proposition ne serait pas complète sans la réorganisation du régime de la compétence lorsque celle-ci concerne les relations avec les États tiers.

\section{B) FORS EXORBITANTS ET RELATIONS AVEC LES ÉTATS TIERS}

La nature même des règles de compétence a pour conséquence que les dispositions d'un règlement européen ne peuvent évidemment ne rendre compétents que les seuls tribunaux européens. A la différence des règles de conflit de lois, dont le caractère universel ne fait aucun doute, les règles de compétence sont nécessairement limitées aux tribunaux des États membres. Il n'en demeure pas moins que cette limite n'exclut nullement d'étendre l'applicabilité des règles de compétence à des situations parfois qualifiées d' " externes », en ce sens qu'elles se localisent au moins partiellement dans des États tiers. La question des liens avec les États tiers est donc d'une particulière difficulté en matière de compétence internationale. ${ }^{28}$

Trois régimes principaux coexistent en la matière.

Le premier, historiquement, est celui de la Convention de Bruxelles et désormais du règlement Bruxelles 1, et consiste à fixer comme critère d'applicabilité principal le domicile du défendeur. Celui-ci, non content d'être une règle de compétence, est aussi la règle qui déclenche l'application même du règlement. Dès lors, si le défendeur est domicilié en Europe, s'appliquent les règles de compétence du règlement, si le défendeur est domicilié hors d'Europe, s'appliquent les règles nationales, en ce compris les règles de compétence exorbitantes, au grand dam des litigants non européens. ${ }^{29}$

On a pu justifier cette règle en estimant qu'il s'agissait avant tout de bâtir un régime juridique propre aux « citoyens européens » afin d'en assurer la protection juridiction-

27 KOHLER, C. L'autonomie de la volonté en droit international privé : un principe universel entre libéralisme et étatisme. Rec. Cours Académie de droit international de La Haye, 2013, vol. 359, spéc. pp. 398 et seq.

28 Sur l'ensemble, v. part. MALATESTA, A. - BARIATTI, S. - POCAR, F. (dir.). The external dimension of EC private international law in family and succession matters. Studi et pubblicazioni della rivista di diritto internazionale privato et processuale, 2008, vol. 71.

29 V. par ex. les critiques de JUENGER, F. A shoe unfit for globetrotting. UC Davis Law Review, 1995, vol. 28, no 3, p. 1027. 
nelle, ou aux litiges « intégrés à l'Union européenne ».30 La justification, pourtant, ne paraît pas absolument convaincante. ${ }^{31}$ Elle suppose une délimitation entre litige européen et litige international qui est presque impossible à tracer a priori et qui, en toute hypothèse, ne peut guère résulter des critères de compétence eux-mêmes. Qualifier ainsi « d'européen » un litige uniquement en raison du domicile ou de la résidence européenne du défendeur n'apparait guère convaincant, tant des litiges peuvent être intégrés à l'ordre juridique de l'Union même lorsque le défendeur est domicilié dans un État tiers. Il est difficile de comprendre, par exemple, qu'une compétence aussi simple que celle fondée sur le lieu d'exécution du contrat puisse être déterminée en application du droit judiciaire de l'Union lorsque le défendeur est domicilié en Europe et du droit national d'un État membre lorsqu'il ne l'est pas. L'opportunité d'un critère de compétence ne varie pas en fonction des rattachements, plus ou moins européens, d'une situation juridique. Aussi d'ailleurs la solution a-t-elle été nuancée pour un certain nombre de chefs de compétence particulièrement importants (compétences exclusives, compétences de protection, clauses attributives de juridiction). La solution du règlement Bruxelles 1, nous semble donc aborder la question complexe des relations avec les États tiers par un biais contestable.

Le second régime est celui du règlement Bruxelles 2 et paraît plus convaincant. ${ }^{32}$ Les articles 6 et 7 du règlement Bruxelles 2 bis (bientôt remplacés par l'article 6 du règlement Bruxelles 2 ter) organisent en matière de divorce une hiérarchie des règles de compétence dont il résulte en substance une double solution. D'une part, si l'un des critères de compétence du règlement permet de rendre compétent le tribunal d'un État membre, la disposition du règlement est applicable à l'exclusion de toute règle nationale, quels que soient par ailleurs les autres éléments de rattachement. ${ }^{33} \mathrm{D}$ 'autre part, les règles nationales sont applicables à défaut de tribunal européen rendu compétent par une disposition du règlement, même si elles ne sont pas opposables aux ressortissants des États membres ou aux personnes domiciliés sur le territoire des États membres. Une telle solution permet donc d'organiser une hiérarchie, sur le modèle de celle qui a été mise en place en France par le célèbre arrêt Cognacs et Brandies de France, ${ }^{34}$ entre les règles de compétence « ordinaires », ici celles du règlement, et les règles de compétence « exorbitantes », ici celles du droit international privé national, les secondes n'étant applicables qu'à défaut des premières.

Cette seconde ligne de solution est un progrès par rapport à la précédente. Elle repose sur un critère de pure compétence qui semble plus rationnel que celui de champ d'application du règlement Bruxelles 1, les compétences exorbitantes restent nationales,

30 Sur ces expressions, v. DROZ, G. Compétence judiciaire et effets des jugements dans le marché commun. Dalloz : Bibliothèque de droit international privé, 1972, Vol. XIII, p. 45 ; GOTHOT, P. - HOLLEAUX, D. La Convention entre les Etats membres de la CEE sur la compétence judiciaire et l'exécution des décisions en matière civile et commerciale. Clunet, 1971, pp. 747 et seq., spéc. p. 755.

31 Pour le détail de l'argumentation sur le règlement Bruxelles 1, on se permettra de renvoyer à PATAUT, E. Qu'est-ce qu'un litige intracommunautaire? Mélanges Normand. Litec, 2003, p. 365.

32 V. aussi, FRANCQ, op. cit., p. 57.

33 En ce sens : CJCE, 29 novembre 2007, aff. C-68/07, Sundelind Lopez ; en France, v. p. ex. Civ. 1, 15 novembre 2017. Rev. Crit, 2018, 581, note C. Chalas.

34 Civ. 1, 19 novembre 1985, Grands arrêts de la jurisprudence française de droit international privé. Dalloz, no 71 . 
mais elles ne sont disponibles qu'au cas où aucun tribunal européen n'est rendu compétent par le règlement. Elle n'en reste pas moins critiquable.

La multiplication de ces règles exorbitantes rend en effet délicate la situation des litigants étrangers domiciliés dans un État tiers. Ceux-ci sont soumis à un ensemble disparate et difficile à connaître de règles exorbitantes, dont l'efficacité est d'autant plus grande qu'elles n'empêchent nullement la décision rendue de circuler automatiquement sur le territoire de tous les autres États membres. En réalité, la difficulté ici n'est pas tant l'existence de règles de compétence exorbitantes, qui peuvent être politiquement justifiées, que leur empilement anarchique. L'unification des règles de compétence est faite en Europe, et s'il faut conserver des compétences exorbitantes pour se protéger contre les justices étrangères, il n'est pas convaincant que la charge de l'organisation de cette protection soit confiée à chaque État membre plutôt que de résulter d'une décision politique commune.

Aussi est-il permis de préférer un troisième modèle, permettant une intégration plus complète : celui qui avait été proposé par le GEDIP en matière civile et commerciale ${ }^{35}$ et qui a été retenu par les règlements en matière de droit patrimonial de la famille. Ce modèle est celui de l'exclusion pure et simple des règles nationales de compétence, qui sont donc entièrement remplacées par les règles européennes. Ainsi par exemple en matière de successions. Ce règlement, en effet, se substitue entièrement au droit national, mesures provisoires et conservatoires mises à part (article 19). Le critère principal, celui de la résidence habituelle du défunt (article 4), se double ainsi, outre d'une possibilité de choix de for (article 5), d'une série de règles de compétence dites « subsidiaires » (article 10) qui permettent de saisir un tribunal européen malgré l'absence de domicile du défunt en Europe (nationalité, lieu de situation des biens...). Aucune place n'est donc laissée aux règles nationales. Les relations avec les États tiers n'en sont pas pour autant oubliées, mais sous la forme de règles particulières, plus ou moins développées selon les règlements.

Telle est bien la voie dans laquelle s'engage résolument le projet du Groupe européen de droit international privé. Les dispositions des articles 4 et suivants ont en effet vocation à remplacer entièrement le droit international privé des États membres. Dès lors, si un tribunal européen n'est pas compétent en application des dispositions du règlement, aucun tribunal européen n'est compétent.

La solution n'interdit pas de prévoir des règles de compétence particulières, éventuellement exorbitantes, résultant d'une politique spécifiquement européenne. Ainsi, notamment, a été incluse dans le texte une compétence fondée sur le «forum necessitatis » (article 9) qui est aujourd'hui suffisamment répandue pour se passer d'explications. ${ }^{36}$

35 V. les « Propositions de modification du règlement 44/2001 en vue de son application aux situations externes », adoptées à la session de Copenhague de 2010, disponibles sur : https://www.gedip-egpil.eu /documents/gedip-documents-20vcf.htm.

36 V. RETORNAZ, V. - VOLDERS, B. Le for de nécessité : tableau comparatif et évolutif. Rev. Crit, 2008, pp. 225-261. Le for de nécessité a été admis dans les règlements Obligations alimentaires (article 7), Successions (article 11) et Régimes matrimoniaux (article 11). 
A été beaucoup plus longuement discutée l'éventuelle introduction d'une règle de compétence résiduelle ou subsidiaire, qui ouvrirait une compétence exceptionnelle en faveur des tribunaux de la nationalité d'un ou des époux sur le modèle du règlement 4/2009 en matière alimentaire (art. 6). Cette règle de compétence particulière aurait constitué une forme de "privilège de citoyenneté européenne ».

Cette solution a finalement été exclue, au profit d'une compétence spécifique figurant à l'article $4 \S 2$, litt. d). L'effet de cette règle est que, si aucun tribunal n'est compétent sur la base de la résidence habituelle des époux ( $\mathrm{a}$ et $\mathrm{b}$ ) ou du défendeur (c), le citoyen européen qui fixe sa résidence habituelle dans un État membre peut saisir les tribunaux de cet État, sans attendre le délai d'un an prévu pour les autres demandeurs.

Il s'agit d'un privilège de citoyenneté, mais qui diffère grandement d'une simple compétence fondée sur la nationalité. D'abord, la compétence ne résulte pas de la seule nationalité, mais de la double condition de la résidence habituelle dans un État membre et de la nationalité. Elle se distingue aussi de l'actuel article 3 du règlement 2019/1111, car elle n'exige pas que le demandeur ait la nationalité de l'État de résidence et, surtout, elle n'est disponible qu'en l'absence de résidence habituelle du défendeur dans l'Union. L'utilisation du for de la nationalité du demandeur est donc beaucoup plus strictement encadrée. La politique proposée vise donc bien à réaliser un équilibre entre la nécessité de ne pas laisser le citoyen européen sans protection, tout en veillant à ne pas empiéter abusivement sur la compétence raisonnable des États tiers.

C'est encore cette préoccupation qui guide la rédaction de l'article 6. Celui-ci vise à traiter de la question de l'élection d'un for d'un État non membre. Son objectif est de parvenir à un équilibre entre l'efficacité de la clause et la compétence des tribunaux de l'Union, en autorisant les tribunaux européens, à certaines conditions, à décliner leur compétence lorsqu'un accord des parties a rendus compétents les tribunaux d'un État tiers. Cette considération de la compétence des États tiers est encore renforcée par l'existence de la règle de litispendance, qui permet, sur le modèle de l'article 33 du règlement Bruxelles 1, de surseoir à statuer et de se dessaisir lorsqu'un tribunal d'un État tiers a été premier saisi (article 13§3). Le projet cherche donc sur ce point une cohérence (encore renforcée, on le verra, par les règles relatives à la circulation des décisions en provenance d'États tiers) dans l'articulation du régime de compétence avec les États tiers.

Il va donc, sur ce point, bien plus loin que l'actuel règlement Bruxelles 2 ter, dont les règles relatives à la litispendance ne concernent que les États membres (article 19) ou encore que le règlement aliments, qui, s'il admet le choix de for (article 4) ne l'envisage, comme la litispendance (article 12), que pour les États membres. De tels choix soulèveront à terme d'importantes difficultés quant au sort à réserver à une clause attributive de juridiction aux tribunaux d'un État tiers.

Comme on le voit, la proposition du GEDIP vise à réformer en profondeur le régime de la compétence en matière de divorce. Une telle voie permettrait, à nos yeux, d'ajouter simplicité et prévisibilité à un contentieux du divorce aujourd'hui d'une excessive et regrettable complexité.

Les modifications proposées sont moins spectaculaires en matière de conflit de lois. 


\section{LES RĖGLES DE CONFLIT DE LOIS}

Sous réserve de ce qui sera discuté quant aux divorces sans juge, les propositions du GEDIP sont beaucoup moins fondamentales en ce qui concerne la loi applicable.

De fait, le règlement Rome 3 reflète un système qui, déjà présent dans plusieurs codifications nationales récentes et repris depuis lors par d'autres règlements, établit une échelle de rattachements en cascade privilégiant la localisation de la résidence habituelle des époux dans le même pays, au nom d'un principe de proximité. Il ouvre également une faculté de choix de loi aux parties. Cette faculté a déjà été discutée et analysée en profondeur, ${ }^{37}$ parfois critiquée. ${ }^{38}$ Il reste que ces critiques ne semblent pas sans réplique, notamment parce que la liberté de choix participe, quoi qu'on en dise, d'un mouvement à la fois de libéralisation du divorce et d'ouverture aux parties de facultés d'anticipation du règlement des conséquences personnelles et pécuniaires de leur union et de son éventuelle dissolution. ${ }^{39}$ L'extension progressive de son empire en matière de droit de la famille inclut désormais le divorce et il n'a pas semblé opportun de revenir sur ce point.

Dès lors, les articles 16 et 17 de la proposition sont largement repris des articles 5 et 8 du règlement 1259/2010, rapprochés pour des raisons de simplicité et faisant l'objet de quelques ajustements techniques.

Le choix de loi, tout d'abord, est assoupli, qu'il porte sur la loi de la nationalité ou sur la loi de la résidence habituelle.

Dans le premier cas, le projet d'article $17 \S 4$ propose de permettre aux époux de choisir la loi de l'une ou l'autre de leur nationalité lorsqu'ils en possèdent plusieurs. Cette solution se distingue de celle du règlement Rome 3 , qui renvoie simplement au droit national dans son considérant $n^{\circ} 22$, à l'instar des règlements sur les régimes matrimoniaux ou patrimoniaux entre partenaires. ${ }^{40}$

La solution semble cohérente avec la faculté de choix qui est ouverte aux parties, qui ne suppose nullement de faire prévaloir une nationalité sur l'autre. La solution du conflit de nationalités, en effet, peut sans difficulté être laissée à la discrétion des parties lorsque le choix de loi ne dépend pas d'un élément objectif mais bien de la volonté de celles-ci. Dans ce cas, en effet, il n'est pas nécessaire que l'ordre juridique du for fasse un choix entre les deux nationalités pour préférer l'une à l'autre, puisque ce choix appartient aux intéressés eux-mêmes. Dès lors, aucune nécessité, ni logique, ni juridique, n'oblige l'État du for à choisir entre les nationalités des époux, qu'il convient au contraire d'investir de la possibilité de choisir entre leurs nationalités. Cette solution est

\footnotetext{
37 KOHLER, Cours précité, spéc. pp. 420 et seq.

38 V. not. MAYER, P. - HEUZE, V. - REMY, B. Droit International Privé. 12 éd. LGDJ, 2019, nº 606.

39 Pour une discussion d'ensemble, v. not. CORNELOUP, S. - JOUBERT, N. Autonomie de la volonté et divorce : le règlement Rome III. Dans : FULCHIRON, H. - PANET, A. - WAUTELET, P. (dir.). L'autonomie de la volonté en droit des personnes et de la famille dans les règlements de droit international privé européen. Bruylant, 2017, p. 179. Sur l'anticipation, v. aussi CHALAS, C. Contrats de mariages et nuptial agreements : vers une acculturation réciproque ? Journal du Droit International, 2016, pp. 781-826.

40 V. les considérants no 50 du règlement 2016/1103 et 49 du règlement 2016/1104.
} 
cohérente avec le projet GEDIP en matière de conflits de nationalités ${ }^{41}$ et, plus positivement, avec le règlement successions, dont l'article 22-1 al. 2 permet lui aussi le choix de loi en faveur des différentes nationalités du de cujus.

L'assouplissement porte aussi sur le choix en faveur de l'une des résidences habituelles des époux, puisque celui-ci peut se porter sur la loi « d'une résidence habituelle commune des époux au cours du mariage » (article $17 \S 1$ litt. a), ce qui est incontestablement plus libéral que la solution actuelle du règlement Rome 3 , qui exige que le choix se fasse ou bien en faveur de la résidence habituelle des époux « au moment de la conclusion de la convention » ou bien en faveur de la « dernière résidence habituelle des époux, pour autant que l'un d'eux y réside encore au moment de la conclusion de la convention » (article $5 \S 1$ ). La solution du règlement Rome 3 a l'avantage de la sécurité et de la prévisibilité, mais l'inconvénient corrélatif de la rigidité. Elle risque notamment, dans le premier cas, de conduire à l'application d'une loi avec laquelle les époux n'ont plus de lien depuis fort longtemps.

Pour y répondre, la souplesse proposée vise à permettre aux époux de se soumettre à une loi avec laquelle ils entretiennent ou ont entretenu un lien étroit et donc de conférer aux époux le soin de choisir entre plusieurs possibilités s'ils ont eu, au cours de leur mariage, plusieurs résidences habituelles. La solution n'exclut certes pas totalement l'inconvénient du règlement Rome 3 : celui de risquer de figer un choix en faveur d'une loi avec laquelle les époux n'ont plus de liens, choix qui, au moment du divorce, sera difficile à remettre en cause par un accord des parties qui ne peut plus être obtenu en raison de la dégradation de leurs rapports. Le risque n'en paraît pas moins atténué et, en donnant une plus grande liberté aux époux, il leur permet de choisir celui des rattachements qu'ils estiment le plus pertinent.

Surtout, pour pallier les risques d'un choix de loi aux conséquences regrettables pour l'une des parties qui aurait consenti, par exemple au moment du mariage, sans peser toute la portée d'un choix, il a été décidé d'introduire une limite à la liberté de choix. Il est en effet proposé de permettre au juge de remettre en cause le choix de loi. L'article $18 \S 3$ du projet affirme ainsi que :

«À moins que les parties n'aient été pleinement informées et conscientes des conséquences de leur choix au moment de la désignation, la loi désignée ne s'applique pas lorsque son application entraînerait des conséquences manifestement inéquitables ou déraisonnables pour l'une ou l'autre des parties ».

La formulation est directement inspirée de l'article $8 \S 5$ du Protocole de La Haye de 2007 sur la loi applicable aux obligations alimentaires ; elle vise à introduire un élément de justice matérielle, laissé entre les mains des juges, compensant ainsi le relatif assouplissement en matière de liberté de choix.

On le voit, si l'équilibre fondamental du texte reste celui du règlement Rome 3, les quelques ajustements réalisés sont loin d'être négligeables.

41 V. 1'article 9 de la position du Groupe européen de droit international privé sur la solution des conflits positifs de nationalités dans les instruments existants de droit international privé de l'Union européenne, adopté à sa session de Lausanne, 2013. Disponible sur : https://www.gedip-egpil.eu/documents/gedip -documents-23.htm. 
Ce sont les mêmes ajustements auxquels il est suggéré de procéder en matière d'ordre public. On sait en effet que la question, délicate entre toutes, des lois prohibitives en matière de divorce a donné lieu à de complexes négociations dans l'Union européenne, expliquant en partie le recours au mécanisme de la coopération renforcée. ${ }^{42}$ Le résultat de ces difficultés a donné lieu à un ensemble complexe et fréquemment critiqué, des articles 10,12 et 13 du règlement, qui prévoient des mécanismes s'apparentant à l'ordre public. ${ }^{43}$

La proposition vise à simplifier radicalement cette difficulté, en prévoyant simplement que la loi du for s'applique lorsque la loi étrangère désignée ne connait pas le divorce (article 21) et en revenant à la formulation traditionnelle de l'ordre public (article 23). La première disposition permet de consacrer ce véritable « droit au divorce » auquel tend désormais l'ordre juridique européen, ${ }^{44}$ la seconde semble suffisante pour s'opposer aux lois qui porteraient une atteinte grave aux règles de l'ordre juridique $\mathrm{du}$ for. L'ordre public est suffisamment large et plastique pour pouvoir s'adapter à de nombreuses difficultés, les tentatives de précisions apportant souvent plus de confusion que de clarté.

En revanche, sous réserve d'une admission limitée du renvoi lorsque celui-ci permet d'appliquer la loi d'un État membre plutôt que celle d'un État tiers (article 22§2), la règle de conflit objective n'a pas fait l'objet de modification et l'article 16 du projet GEDIP est le décalque de l'article 8 du règlement Rome 3.

On le voit, ajustements et non bouleversements. C'est un peu la même chose en matière de reconnaissance des décisions, à l'importante nuance près du régime de reconnaissance des décisions rendues dans des pays tiers.

\section{LA RECONNAISSANCE DES DÉCISIONS}

La proposition de modification la plus fondamentale en matière de reconnaissance des décisions ne concerne pas les décisions rendues dans un État membre. Le règlement Bruxelles 2 ne concernant que le principe du divorce, aucune disposition relative à l'exécution n'est nécessaire. Le régime actuel est donc un régime de reconnaissance simple, libéral et peu contesté. Le principe fondamental en est la reconnaissance automatique (article 30), qui permet de se prévaloir de la dissolution du lien matrimonial sans procédure particulière dans tous les États membres. Cette reconnaissance automatique permet par exemple la transcription d'un divorce sur les registres d'état civil sans procédure particulière (article 30-2).

La reconnaissance, bien sûr, peut être discutée, par les voies habituelles de la reconnaissance incidente, ou de l'action en opposabilité ou en inopposabilité (articles 30 et 59 à 62 ). Si une telle procédure est déclenchée, les conditions de contrôle sont

42 HAMMJE, P. Le nouveau règlement (UE) n $n^{0}$ 1259/2010 du Conseil du 20 décembre 2010 mettant en œuvre une coopération renforcée dans le domaine de la loi applicable au divorce et à la séparation de corps. Revue Critique de Droit International privé, 2011, pp. 291-338, no 1 et 13.

43 Ibid., no 41 et seq.

44 V. la discussion dans HAMMJE, P. Divorce et séparation de corps. Répertoire Dalloz de droit international, 2018, no 205. 
peu nombreuses, puisque, en substance, seul l'ordre public, de fond ou procédural, ou l'inconciliabilité de décisions peut conduire au refus de reconnaissance. On sait que ce régime est extrêmement libéral, ne permettant que très exceptionnellement un tel refus de reconnaissance. La Cour a, encore récemment, rappelé sa conception très restrictive de l'ordre public, qui n'empêche pas la circulation de la décision, même lorsque le juge qui l'a rendu a violé les dispositions du règlement sur la litispendance, en refusant de se dessaisir, quoique second saisi. ${ }^{45}$

Aucune justification ne paraît devoir conduire à remettre en cause cette orientation libérale et favorable au divorce. Aussi le texte proposé par le GEDIP, au-delà de quelques modifications de langage, propose-t-il de reconduire purement et simplement le régime de reconnaissance des décisions. Les motifs de refus de reconnaissance, tout particulièrement, sont les mêmes (article $28 \mathrm{du}$ projet).

L'innovation principale est ailleurs. La proposition suggère en effet d'inclure un régime complet de reconnaissance des décisions en provenance des États tiers. Il ne s'agit pas d'une nouveauté pour le GEDIP, qui s'est déjà prononcé en faveur d'un régime commun de reconnaissance des décisions rendues dans des États tiers. La solution avait été proposée en matière civile et commerciale aux sessions de Padoue (2009) et Copenhague (2010) ${ }^{46}$ Le principe de cette inclusion est évidement susceptible d'être contesté, notamment sous l'angle de la compétence de l'Union. En 2010, il avait toutefois été estimé que l'obstacle n'était pas dirimant, dans la mesure d'une part où la disparité des règles de reconnaissance pouvait conduire à d'insurmontables difficultés de fonctionnement du marché intérieur en cas de divergences entre États membres relativement à la reconnaissance ou l'exécution d'une décision et, d'autre part, où l'avis $1 / 03$ sur la compétence externe de l'Union sur la Convention de Lugano ${ }^{47}$ conférait bien une compétence à l'Union en la matière. ${ }^{48}$

Depuis, l'évolution des textes n'a fait que confirmer cette analyse ; il n'est que de songer au règlement Bruxelles 1 bis qui a commencé, certes timidement, à réglementer certains des aspects des relations avec les États tiers. ${ }^{49}$

En outre, et même si, comme on le verra, le règlement Bruxelles 2 ter n'a pas suivi cette ligne, l'inclusion de divorces non juridictionnels dans le domaine d'un régime de circulation des divorces sans tenir compte de ceux obtenus dans des États tiers serait problématique. En raison, précisément, de leur nature privée, la ligne de démarcation entre les divorces privés « européens » et les divorces privés « non européens » risque de poser de nombreuses difficultés, rendant bien moins intéressantes et opératoires les solutions proposées pour faciliter la circulation de ces divorces.

Ces arguments ont convaincu et conduit à inclure dans la proposition un régime complet de reconnaissance des décisions de divorce rendues dans les États tiers.

45 CJUE, 16 janvier 2019, aff. C-386/17, Liberato.

46 V. la « Proposition de modification du chapitre II du règlement 44/2001 en vue de son application aux situations externes » adoptée à la session de Copenhague. Disponible sur : https://www.gedip-egpil.eu /documents/gedip-documents-20vcf.htm.

47 CJUE, Assemblée Plénière, avis du 7 février 2006, 1/03.

48 FALLON, M. Commentaire de la proposition. Disponible sur : https://www.gedip-egpil.eu/documents /gedip-documents-20cf.htm.

49 Ainsi par exemple de la litispendance, v. l'article 33 et les considérants 23 et 24 du règlement. 
En ce qui concerne le régime de cette reconnaissance, le texte proposé pour les décisions de divorce rendues dans les États tiers est, donc, inspiré des propositions adoptées par le Groupe lors de ces précédentes sessions.

Celui-ci est calqué sur celui des décisions rendues dans les États membres, notamment sur le point essentiel de la reconnaissance de plein droit. Aucune procédure préalable n'est donc, ici encore, nécessaire pour procéder à une transcription sur les registres de l'état civil d'une décision de divorce obtenue dans un État tiers (article 36).

Les différences importantes portent plutôt sur les conditions de reconnaissance en cas de contestation. Les exigences de contrôle de l'ordre public et d'inconciliabilité sont maintenues. Mais la rigueur du contrôle est doublement renforcée.

On notera tout particulièrement le maintien à l'article $37 \S 1$ d'une forme de contrôle de la compétence du juge d'origine, sous la forme d'un contrôle de l'étroitesse des liens entre le juge d'origine et le divorce, sur le modèle que connaissent déjà certains Etats européens, ${ }^{50}$ mais pas tous, loin s'en faut. ${ }^{51}$ La généralisation d'une telle condition participerait donc bien d'une libéralisation des mécanismes de reconnaissance.

La fraude, en revanche, n'est pas retenue, mais elle n'est pas étrangère à l'adoption d'une disposition, plus objective, de conflit de procédures : une décision étrangère qui aurait été rendue avant une décision européenne antérieure ne pourrait pas être reconnue si le tribunal européen avait été saisi en premier lieu (art. 37§2). La précision doit être lue comme le pendant de la règle de litispendance qui autorise les juges européens à surseoir à statuer lorsqu'ils sont saisis en second et que la décision étrangère fait l'objet d'un pronostic de reconnaissance favorable (article 13). Dans la situation inverse, la priorité de compétence revendiquée par le tribunal européen se traduit, si elle n'est pas respectée, par un barrage à la reconnaissance de la décision étrangère qui l'aurait violée.

On le voit, le système de reconnaissance n'est pas, en lui-même, révolutionnaire. Il a en revanche l'avantage d'être libéral, raisonnablement simple et, surtout, exhaustif, ce qui permet aussi de régler une importante difficulté relative à la reconnaissance des divorces sans juge.

\section{LE DIVORCE SANS JUGE}

Les modes de dissolution du mariage évoluent rapidement en Europe. Alors, en effet, que la dissolution judiciaire était manifestement la seule à l'esprit des rédacteurs des règlements Rome 3 et Bruxelles 2, la difficulté posée par les divorces obtenus par actes privés s'est progressivement imposée dans le débat.

La question, historiquement, concerne avant tout les divorces obtenus hors d'Europe et, plus particulièrement, les répudiations musulmanes. La difficulté était d'abord méthodologique : s'agissant de divorces dépendant fondamentalement de la volonté des parties, il était permis d'hésiter entre la méthode du conflit de lois, suivie par certains

50 V. not. en droit français, le célèbre arrêt Simitch de la Cour de cassation : Civ. 1, 6 février 1985, Rev. Crit. DIP, 1985 , p. 369.

51 V. les remarques comparatives de CUNIBERTI, G. Le fondement de l'effet des jugements étrangers. Recueil des Cours, 2018, vol. 394, pp. 93 et seq., spéc. n 77, p. 146. 
pays, ${ }^{52}$ et celle de la reconnaissance des décisions, suivie par d'autres. ${ }^{53}$ La même question se pose désormais pour les divorces privés acceptés dans certains pays d'Europe, avec d'autant plus d'acuité que le législateur national s'est parfois purement et simplement désintéressé des aspects internationaux de ceux-ci. ${ }^{54}$

Le débat ne pouvait donc manquer de se déplacer du droit national vers le droit de l'Union, au fur et à mesure de l'européanisation du droit international privé du divorce. C'est bien ce qui a eu lieu, lorsque, la première, la Cour de justice, a été saisie de la difficulté. 55

L'affaire Sahyouni illustre à merveille les enjeux méthodologiques propres aux divorces privés et les difficultés à tracer en la matière une ligne entre les divorces « européens » et les autres. Il s'agissait en effet d'un divorce obtenu en Syrie par un couple dont les deux époux possédaient la double nationalité, syrienne et allemande, et dont le domicile avait été alternativement en Allemagne, en Syrie et dans d'autres pays arabes.

En 2013, le mari a déclaré vouloir divorcer de son épouse et son représentant a prononcé la formule de divorce devant le tribunal religieux de la charia de Latakia (Syrie). Le 20 mai 2013, ce tribunal a constaté le divorce des époux.

L'épouse s'opposant à la reconnaissance du divorce en Allemagne, il convenait de savoir si le droit de l'Union était applicable et, si oui, quel texte. Cette première question avait d'ailleurs conduit à une première décision d'incompétence de la Cour de justice, celle-ci n'étant pas convaincue de l'application d'un texte de droit de l'Union à un divorce obtenu dans un État tiers. ${ }^{56} \mathrm{Il}$ ne faisait aucun doute, en effet, que le règlement Bruxelles 2 n'était pas applicable. L'applicabilité du règlement Rome 3, en revanche, était plus discutable. Telle était pourtant bien la position du droit allemand, reprise par la Cour :

«En particulier, il ressort des informations fournies par cette juridiction ainsi que des observations du gouvernement allemand que, en vertu du droit allemand, la reconnaissance des divorces prononcés dans un État tiers est effectuée dans le cadre de la procédure prévue à l'article $107 \mathrm{du}$ FamFG. Conformément à cette disposition, la reconnaissance des décisions d'une juridiction ou d'une autorité étatiques étrangères prononçant un divorce de manière constitutive est accordée en l'absence de tout examen de leur légalité, alors que la reconnaissance des divorces privés est subordonnée au contrôle de leur validité au regard du droit matériel de l'État désigné par les règles de conflit de lois pertinentes $\gg$ ( $\mathrm{n}^{\circ} 30$, c'est moi qui souligne).

Le droit allemand subordonnait donc l'efficacité en Allemagne d'un divorce privé étranger au respect de la loi qui lui aurait été applicable en droit allemand, laquelle s'établissait, au moment du litige, par application de la règle de conflit de lois issue du

52 Sur le débat en Allemagne, v. part. GRUBER, U. P. Scheidung auf Europäisch - die Rom III-Verordnung. Praxis des Internationalen Privat und Verfahrensrechts, 2012, p. 381.

53 EL-HUSSEINI, R. Le droit international privé français et la répudiation islamique. Revue Critique de Droit International Privé, 1999, pp. 427 et seq. V. en Belgique l'article 57 de la loi du 16 juillet 2004 portant le Code de droit international privé. Revue Critique de Droit International Privé, 2005, pp. 154-226.

54 Sur le débat français, v. part. HAMMJE, P. Le divorce par consentement mutuel extrajudiciaire et le droit international privé. Les aléas d'un divorce sans for. Revue Critique de Droit International Privé, 2017, p. 143.

55 CJUE, 20 décembre 2017, aff. C-372/16, Sahyouni 2.

56 CJUE, 12 mai 2016, aff. C-281/15, Sahyouni 1. 
règlement Rome $3 .{ }^{57} \mathrm{La}$ question posait donc bien une difficulté d'interprétation du droit de l'Union.

Au rebours du droit allemand, la Cour a toutefois décidé que le règlement n'était pas applicable, les divorces privés restant en dehors de son champ d'application. La décision a incontestablement suscité quelques difficultés, dont la moindre n'était pas la résurrection de règles de conflit dont on pensait parfois qu'elles étaient sorties du droit positif. ${ }^{58}$ Elle n'en posait pas moins l'importante difficulté des liens entre divorces privés et droit de l'Union, question d'autant plus brûlante que, précisément, les divorces privés faisaient progressivement leur apparition en Europe.

Aussi faut-il se féliciter que le règlement Bruxelles 2 ter ait proposé une solution en la matière. .9

Il n'est pas certain, toutefois, que celle-ci soit entièrement satisfaisante.

Le règlement contient tout d'abord une définition de l'accord à l'article 2 ; cette définition est ensuite complétée par une section nouvelle, consacrée aux « actes authentiques et accords » (articles 64 et suivant). La règle concernant les accords est méthodologiquement proche d'une règle de reconnaissance des décisions, puisque l'article 65 renvoie, pour les accords et les actes authentiques, aux dispositions générales gouvernant la reconnaissance des décisions. La solution est encore explicitée par le considérant $\mathrm{n}^{\circ} 70$, aux termes duquel :

«Les actes authentiques et les accords entre parties relatifs à la séparation de corps et au divorce qui ont un effet juridique contraignant dans un État membre devraient être assimilés à des 'décisions' aux fins de l'application des règles de reconnaissance ».

La ratio legis ne fait guère de doute, donc, et il s'agit bien d'une règle de reconnaissance, fort libérale, des divorces privés. Il reste toutefois à régler la difficulté des relations avec les États tiers. Une décision privée n'étant pas, par nature, aisément localisable et les autorités des États membres n'étant pas gouvernées par des règles de compétence internationale identiques à celle des juges, la difficulté est de taille.

Pour la contourner, le nouveau règlement procède, là encore, par détermination d'un champ d'application spécifique au règlement. Il faut d'une part que l'accord ait été « enregistré » par une autorité publique dans un État membre (article 2§3). Mais, d'autre part et surtout, les dispositions nouvelles relatives à la reconnaissance ne sont applicables qu'aux actes et accords qui ont été enregistrés ou dressés « dans un État membre dont les juridictions sont compétentes au titre du chapitre II » (article 64).

Cette dernière condition est bien une disposition de champ d'application en ce sens que si cette condition n'est pas remplie, les règles relatives à la reconnaissance ne seront tout simplement pas applicables. En d'autres termes, lorsque l'accord aura été ou bien conclu dans un État tiers ou bien conclu dans un État membre, mais dans une hypothèse où les juridictions de cet État membre n'auraient pas été compétentes si elles avaient été saisies du divorce, les nouvelles règles relatives à la reconnaissance ne s'applique-

57 Sur ces points, v. le commentaire de l'arrêt Sahyouni 2 par COESTER-WALTJEN, D. Praxis des internationalen Privat- und Verfahrensrechts, 2018, p. 238.

58 Sur le droit français et l'inopportune résurrection de l'article 309 du Code civil, v. le commentaire de la décision par P. HAMMJE, P. Revue Critique de Droit International Privé, 2018, pp. 902 et seq.

59 V. la présentation critique exhaustive de FRANCQ, op. cit., pp. 69 et seq. 
ront pas. Il faudra donc nécessairement, dans ce cas, en revenir au droit international privé du for. Dans la mesure où les autorités en question (avocats, notaires ou autorités publiques) ne sont pas nécessairement gouvernées par des règles de compétence, l'hypothèse n'est nullement d'école.

Dès lors, si la solution est incontestablement habile, elle a le défaut de laisser en dehors de son champ d'application non seulement des divorces privés étrangers, mais encore des divorces privés européens.

C'est cette regrettable issue qu'a tenté d'éviter le projet avancé par le Groupe européen de droit international privé, dont les articles $3,40,41$ et 42 sont consacrés à cette question.

La difficulté première tenait à la définition même des divorces privés, qui est malaisée. La solution a consisté à distinguer tout d'abord entre les divorces « juridictionnels » et les divorces «non juridictionnels », tels que définis dans l'article 3 du projet. L'article $3 \S 1$ s'inspire très directement de la formulation de l'article $3 \S 2$ du règlement 2016/1103 sur les régimes matrimoniaux et vise à englober dans sa définition non seulement les divorces prononcés par un juge, au sens étroit et classique du terme, mais encore par une autorité qui aurait en la matière des prérogatives équivalentes et, surtout, dont la décision pourrait faire l'objet d'un recours. L'article $3 \S 2$, pour sa part, renvoie à toutes les formes de divorce obtenues sans le concours de cette juridiction.

Cette définition étant posée, reste à en déterminer le régime juridique de reconnaissance, qui vise, on le rappelle, les divorces obtenus dans les États membres et ceux obtenus dans les États tiers.

Le point principal est d'assimiler purement et simplement les divorces « reçus par une juridiction » au sens de l'article $3 \S 1$ aux décisions (article 40) et, en revanche, de rester plus proche de la méthode du conflit de lois pour les divorces purement privés (articles 41 et 42).

L'article 40 vise ainsi les divorces « juridictionnels » basés sur un acte authentique ou un accord. Pourvu qu'ils aient fait l'objet de l'intervention d'une juridiction au sens de l'article $3 \S 1$, ce qui suppose en particulier qu'ils soient dotés d'une force équivalente à celle d'une décision selon le droit de l'État d'origine, ces divorces suivent le régime des décisions.

En revanche, d'autres formes de divorce issues d'un acte ou d'un accord ne répondant pas aux conditions de l'article $3 \S 1$ (et visées à l'art. $3 \S 2$ ) ne sont pas purement et simplement assimilées aux décisions. Elles relèvent au moins en partie de la méthode du conflit de lois en ce qu'elles vont être soumises aux exigences d'une vérification de loi appliquée, combinée avec un contrôle d'ordre public et d'inconciliabilité.

Il paraissait toutefois difficile de condenser le régime de reconnaissance de ces décisions en une seule disposition. Il est apparu, en effet, que des modèles différents, posant des difficultés différentes, pouvaient être isolés, selon que le divorce était consensuel ou, au contraire, purement unilatéral.

Le premier cas vise un contrat ou un acte consensuel enregistré d'une façon ou d'une autre, par une autorité administrative (tel l'officier de l'état civil ou un notaire dans plusieurs États membres, comme la Lettonie ou le Danemark, mais aussi le nouveau divorce consensuel français). Ces divorces purement consensuels doivent avoir 
été conclus en conformité de la loi de nationalité ou de la loi de la résidence d'un époux pour pouvoir être efficaces sur le territoire des États membres. La solution retenue retient donc les rattachements de l'option de droit, tout en étant plus flexible, notamment en ce qu'elle accepte que soit appliquée la loi de la résidence habituelle d'une seule partie.

Ils doivent en outre ne pas être contraires à l'ordre public ni inconciliables avec une décision ou un autre accord. La formulation des règles sur l'inconciliabilité est adaptée des règles classiques, et notamment du nouvel article 68 du règlement 2019/1111. La formulation vise à rendre compte de la possible divergence entre un accord et une décision ou un autre accord.

Le second cas porte sur le divorce unilatéral (tel le talak en droit islamique) et la solution proposée est plus rigoureuse. Celui-ci, pour pouvoir circuler, doit répondre à deux conditions. D'une part, il doit avoir été prononcé en conformité avec la loi applicable telle que déterminée par le règlement et d'autre part, avoir été prononcé dans un État dont la loi admet ce mode de divorce. Cette double vérification permet à la fois de contrôler la loi appliquée (y compris, en vertu de l'art. 23, la conformité à l'ordre public de celle-ci) et de s'assurer qu'un tel divorce purement unilatéral n'a pas été prononcé dans un État tiers qui ne l'admettrait pas (ainsi, par exemple d'un talak prononcé en Suisse ou en Australie entre époux dont la loi nationale commune l'admettrait).

En outre, les divorces purement unilatéraux doivent avoir été acceptés sans équivoque par l'autre partie pour pouvoir être reconnus, sauf exception. Une exception possible, que vise la fin de la phrase du paragraphe 1, est celle de l'écoulement d'un temps relativement long depuis le prononcé de la dissolution. La réalité de la vie familiale pourrait dans ce cas conduire à estimer que ce divorce prononcé en application de la loi compétente est valable en Europe. La formulation essaie de rendre compte de cette situation, pour le cas où la portée du critère de l'intensité de rattachement (Inlandsbeziehung) inhérent à l'appréciation de l'exception générale d'ordre public ne s'avérerait pas suffisamment précise. En revanche, ce critère peut aider à apprécier la reconnaissance d'un tel divorce prononcé à un moment où l'un des époux résidait dans un pays qui ne connaît pas cette forme de dissolution du mariage.

Les règles relatives à la contrariété de décisions, enfin, sont les mêmes que celles de l'article 41.

L'ensemble, comme on le voit, vise à l'exhaustivité. Il est en tout cas beaucoup plus large, à défaut d'être tout aussi libéral, que le nouveau règlement Bruxelles 2 ter. A ce titre, il constitue à tout le moins une base de débat qui permettrait de tenir compte non seulement de l'évolution des droits internes européens, qui s'ouvrent progressivement aux divorces privés, mais encore de la réserve que peuvent inspirer certains divorces purement unilatéraux, dont on sait les importants débats auxquels ils ont donné lieu dans tous les pays européens.

$$
* * *
$$

Les litiges internationaux en matière de divorce sont des litiges souvent âpres, humainement douloureux et d'une insondable complexité juridique. La codifica- 
tion progressive du droit international privé de la famille en Europe a le mérite d'offrir aux parties un cadre juridique ferme. Les règlements européens n'en recèlent pas moins de redoutables chausse-trappes et difficultés, qui permettent de penser que les choses sont encore largement améliorables. C'est ce qu'a tenté de faire, à son modeste niveau, le projet du GEDIP et la codification globale du divorce international qui y est suggérée.

Professor Etienne Pataut

Sorbonne Law School (University Paris 1)

Etienne.pataut@univ-paris1.fr 